\title{
Epidemiological Characteristics and Drug Resistance Analysis of Multidrug-Resistant Acinetobacter baumannii in a China Hospital at a Certain Time
}

\author{
MEIJIE JIANG ${ }^{1}$, ZHIJUN ZHANG ${ }^{2}$ and SHUPING ZHAO ${ }^{1 \star}$ \\ ${ }^{1}$ Department of Clinical Laboratory, The Central Hospital of Taian \\ Taian, Shandong 271000, People's Republic of China \\ ${ }^{2}$ Taishan Medical University, Taian, Shandong 271016, People’s Republic of China
}

Submitted 21 February 2014, revised 7 May 2014, accepted 13 June 2014

\begin{abstract}
Multidrug-resistant Acinetobacter baumannii is an important bacterium causing nosocomial infections; A. baumannii infections have increased in our hospital since 2009. However, multidrug-resistant $A$. baumannii, which was mainly isolated from patients in each intensive care unit (ICU), rapidly increased from December 2012 to January 2013. Therefore, we described the molecular characteristics of A. baumannii by pulsed-field gel electrophoresis (PFGE). We also detected resistance genes for $\beta$-lactam, aminoglycosides, and plasmid-mediated quinolones. Disinfectant-resistant genes were also detected in the clinical isolates of $b_{a_{\text {OXA-51 }}}$-positive multidrug-resistant $A$. baumannii. The conjugative test was performed to detect whether or not resistance genes can be transferred to different strains. Carbonyl cyanide $\mathrm{m}$-chlorophenylhydrazone (CCCP) inhibition test was conducted to analyze the factors influencing the resistance of A. baumannii to imipenem, meropenem, ceftazidime, levofloxacin, and tigecycline. PFGE profiles contained 12 strains, including 20 type C strains (47.6\%), 4 type D strains (9.5\%), and 1 to 3 strains of other types; 38 strains were distributed in patients in each ICU. In our test samples, the presence of bla $a_{\text {OXA-23 }}$ was closely related to carbapenem resistance. The $16 \mathrm{~S}$ rRNA methylase gene armA was associated with resistance to amikacin, gentamicin, and tobramycin. The multidrug-resistant $A$. baumannii was closely related to various resistance genes. These results indicated that multidrug-resistant $A$. baumannii with type $\mathrm{C}$ strains was predominant in our hospital in this period.
\end{abstract}

Key words: Acinetobacter baumannii multiple-drug resistance, pulsed-field gel electrophoresis, resistance gene

\section{Introduction}

Acinetobacter baumannii has emerged worldwide as an important pathogenic bacteria causing nosocomial infections. Because of its extensive drug-resistance, high-clinical infection rate and poor patient outcomes, the emergence of multidrug-resistant and extensively drug-resistant A. baumannii has brought great difficulties to clinical treatment.

The bacteria drug-resistance surveillance of our hospital showed that there were only 13 strains of imipenem-resistant A. baumannii in 2008. However, these increased to 91 strains in 2009. We found that A. baumannii infections have increased in our hospital since 2009 especially from December 2012 to January 2013, during which time multidrug-resistant A. baumannii were mainly isolated from patients in each intensive care unit. To improve the cure rate of patients infected by multidrug-resistant A.baumannii and to prevent a potential $A$. baumannii epidemic in the hospital, we studied the epidemiological characteristics and drug-resistance of nosocomial multidrug- resistant A. baumannii separated from December 2012 to January 2013.

Taian Central Hospital is a general teaching hospital with 1906 beds. This hospital is equipped with the following: an emergency intensive care unit (ICU) with 12 beds, where the number of patients in each month ranges from 80 to 100; an ICU ward with 28 beds, where the number of patients in each month ranges from 70 to 90; a respiratory ICU with 12 beds, where the number of patients in each month ranges from 21 to 37; a neurosurgical ICU with 16 beds, where the number of patients in each month ranges from 60 to 70 ; and neurology ICU with 13 beds, where the number of patients in each month ranges from 55 to 65 .

\section{Experimental}

Materials and Methods

Strain source. A total of 42 multidrug-resistant A. baumannii strains were isolated from inpatients from December 2012 to January 2013. Among these strains,

\footnotetext{
* Corresponding author: Shuping Zhao, Department of Clinical Laboratory, The Central Hospital of Taian, 29 Longtan Street, Taian, Shandong 271000, People’s Republic of China; phone: +86-538-6298372; e-mail: dczhshp@126.com
} 
39 were isolated from sputum, 1 strain was isolated from puncture fluid, 1 strain was isolated from secretions, and 1 strain was isolated from urine.

Bacterial identification and drug sensitivity test. Bacterial identification and drug sensitivity test were performed using a WalkAway 96 PLUS NC50 composite plate. The clinical isolates of $A$. baumannii resistant to cotrimoxazole, ciprofloxacin, levofloxacin, ceftriaxone, ceftazidime, cefepime, ticarcillin/clavulanic acid, and piperacillin/tazobactam were selected. A total of 42 multidrug-resistant $A$. baumannii strains were isolated during this period; among these strains, 38 were isolated from the patients in the ICU.

Detection of drug resistance genes. Bacterial DNA was extracted using the boiling method and polymerase chain reaction (PCR). The beta lactam resistance-related gene, aminoglycoside resistance gene, plasmid-mediated quinolone resistance gene, and qacE $\Delta 1$ primers were characterized according to previously described methods (Shen et al., 2008; Hu et al., 2008; Zhi et al., 2005; Yang et al., 2008; Wang et al., 2009; Yang et al., 2011; Park et al., 2006). The PCR amplification primer sequences of the $b l a_{\mathrm{NDM}-1}$ gene were characterized according to the Chinese Center for Disease Control and Prevention of Infectious Disease Control and Prevention.

DNA sequencing. Positive PCR gene products were sequenced by Shanghai Sunny Biotechnology Co., Ltd. (China); the sequencing result query was verified in the GenBank network.

Homology analysis of the strains. Using pulsedfield gel electrophoresis (PFGE), we encoded and processed the PFGE image in BioNumerics (Version 5.1, AppliedMaths, Inc.). Image strips were obtained using uniform molecular mass standards. The band position was calibrated. Manual correction was performed to analyze data if necessary and 90\% homology was used as threshold to determine the type of strain.

The plasmid conjugation test was performed according to a previously described method (Zhang et al., 2008a), in which Escherichia coli J53 was used as a recipient strain and multidrug-resistant $A$. baumannii clinical isolates were used as donor strains. The identification and drug sensitivity test of the zygote was performed using WalkAway 96 PLUS NC50 composite plate. The three screening media were selected and respectively contained $100 \mu \mathrm{g} / \mathrm{ml}$ sodium azide $+0.5 \mu \mathrm{g} / \mathrm{ml}$ imipenem, $100 \mu \mathrm{g} / \mathrm{ml}$ sodium azide $+2 \mu \mathrm{g} / \mathrm{ml}$ gentamicin, and $100 \mu \mathrm{g} / \mathrm{ml}$ sodium azide $+2 \mu \mathrm{g} / \mathrm{ml}$ amikacin.

Active efflux inhibition test. The 42 multidrugresistant $A$. baumannii strains were analyzed with the carbonyl cyanide $m$-chlorophenylhydrazone (CCCP) inhibition test to detect active efflux in the presence of imipenem, meropenem, ceftazidime, levofloxacin, and tigecycline. $\mathrm{MH}$ agar plates with and without $50 \mu \mathrm{mol} / \mathrm{LCCCP}$ imipenem, meropenem, ceftazidime, and tigecycline concentration gradients (from $256 \mu \mathrm{g} / \mathrm{ml}$ to $0.125 \mu \mathrm{g} / \mathrm{ml}$, double dilution) were prepared to determine bacterial Minimal Inhibitory Concentration (MIC). MH agar plates with and without $50 \mu \mathrm{mol} /$ LCCCP levofloxacin concentration gradient (from $32 \mu \mathrm{g} / \mathrm{ml}$ to $0.016 \mu \mathrm{g} / \mathrm{ml}$, double dilution) were also prepared to determine bacterial MIC. The susceptibility to imipenem, meropenem, ceftazidime, levofloxacin, and tigecycline increased four times after these antibiotics were placed in an efflux pump inhibitor, indicating the presence of a bacterial antibiotic efflux mechanism (Wang et al., 2012).

\section{Results}

Antimicrobial susceptibility test results. The antimicrobial susceptibility of the 42 multidrug-resistant A. baumannii strains to 17 kinds of antimicrobials is shown in Table I.

PFGE results. The PFGE image of the 42 multidrug-resistant $A$. baumannii strains was encoded in BioNumerics for processing and 90\% homology was used as threshold to distinguish the types. The PFGE profiles contained 12 strains (Fig. 1.), including 20 type $\mathrm{C}$ strains (47.6\%) and 4 type $\mathrm{D}$ strains (9.5\%). The remaining types contain 1 to 3 strains, respectively. The spectral pattern showed that these strains were distributed in ICUs, and the distribution of each type in each unit is shown in Table II.

Detection of $\boldsymbol{\beta}$-lactam resistance-related genes and gene sequencing results. bla $a_{\text {OXA-51 }}(100 \%)$ genes were positive in 42 multidrug-resistant $A$. baumannii strains as well as in 41 strains $(97.6 \%)$ containing the bla $a_{\text {OXA23 }}$ gene, 42 strains $(100 \%)$ containing the bla ${ }_{\text {OXA64 }}$ gene, 42 strains $(100 \%)$ containing the bla $a_{\text {OXA64 }}$ set of genes, and 35 strains $(83.3 \%)$ containing the $b l a_{\text {TEM }}$ gene. The positive membrane protein gene of carO was also observed. PCR gene-amplified products of six strains with positive OXA-23 were sequenced. The results showed that these strains contained $b l a_{\text {OXA-23 }}$ genes and the homology with the encoding JN207493 sequence was 100\%. PCR gene-amplified products of eight strains with positive OXA-64 group were sequenced; these strains contained $b l a_{\text {OXA-66 }}$ genes and the homology with the encoding FJ360530 sequence was 100\%. PCR geneamplified products of four strains with positive OXA51 were sequenced; these strains contained bla $a_{\text {OXA-51 }}$ genes and the homology with the encoding JX305944 sequence was $100 \%$. PCR gene-amplified products of three strains positive for TEM were sequenced; these strains contained $b l a_{\text {TEM-1 }}$ genes and the homology with the encoding EF035598 sequence was 100\%.

Aminoglycoside resistance-related genes, plasmid-mediated quinolone resistance genes, and qacE $\Delta 1$ 


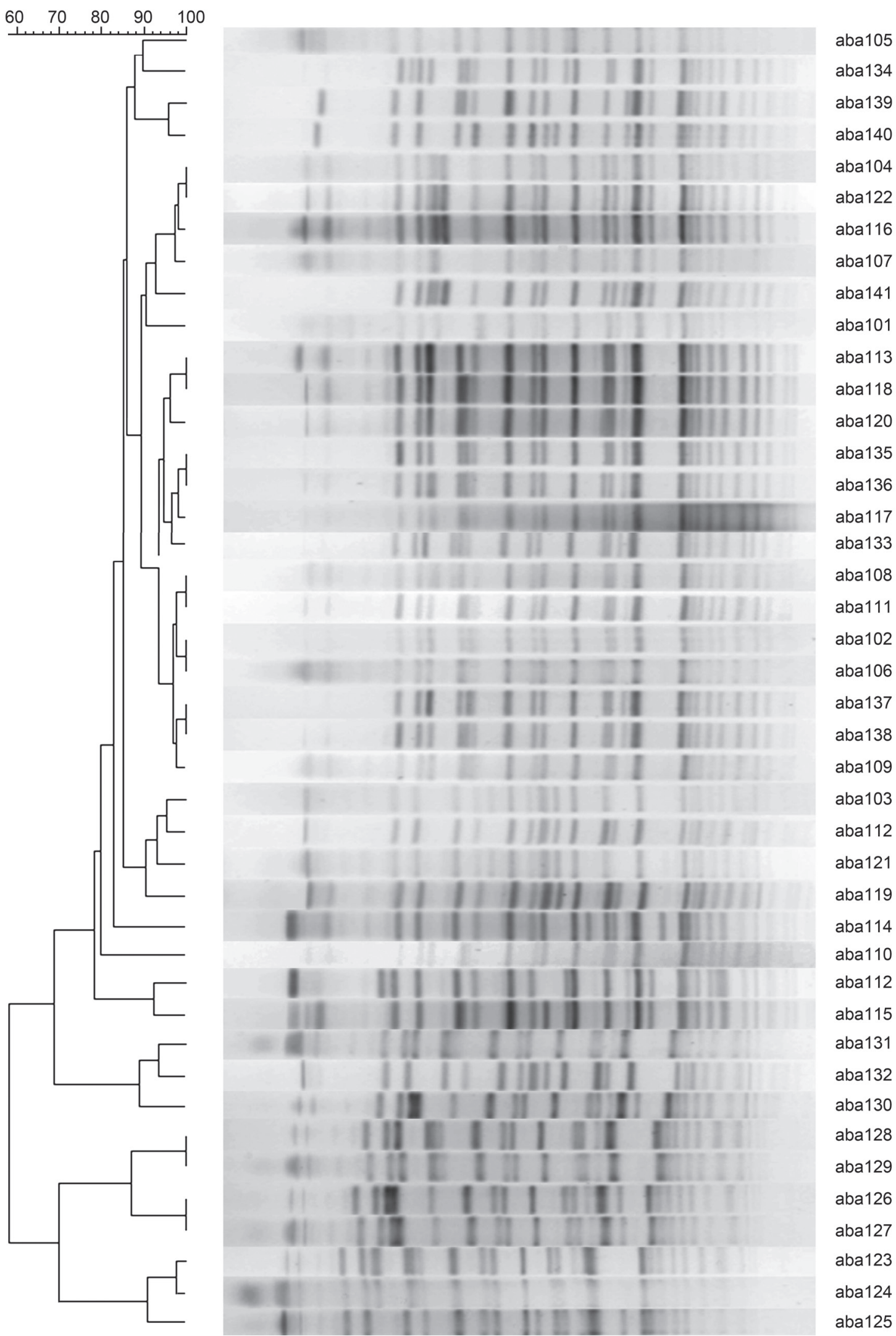

Fig. 1. The PFGE profiles contained 12 strains, including 20 type $\mathrm{C}$ strains (47.6\%) and 4 type D strains (9.5\%). The remaining types contain 1 to 3 strains, respectively.

gene detection and sequencing results. Among the 42 multidrug-resistant $A$. baumannii strains, 41 (97.7\%) contained ant (3")-I gene, 34 (81\%) contained aac (3)-I gene, 7 (16.7\%) had aac (6')-I gene, 16 (38.1\%) contained $\operatorname{armA}$ gene, and $41(97.6 \%)$ carried qacE $\Delta 1$ resistance gene. PCR gene-amplified products of three 
Table I

Antimicrobial susceptibility of the 42 multidrug-resistant Acinetobacter baumannii strains to 17 kinds of antimicrobials

\begin{tabular}{|l|c|c|c|c|c|c|}
\hline \multirow{2}{*}{ Antimicrobial drugs } & \multicolumn{2}{|c|}{ Sensitive } & \multicolumn{2}{c|}{ Intermediary } & \multicolumn{2}{c|}{ Resistant } \\
\cline { 2 - 7 } & $\begin{array}{c}\text { Number } \\
\text { of Strains }\end{array}$ & $\begin{array}{c}\text { Sensitivity } \\
\text { rate }\end{array}$ & $\begin{array}{c}\text { Number } \\
\text { of Strains }\end{array}$ & $\begin{array}{c}\text { Intermediary } \\
\text { rate }\end{array}$ & $\begin{array}{c}\text { Number } \\
\text { of Strains }\end{array}$ & $\begin{array}{c}\text { Resistance } \\
\text { rate }\end{array}$ \\
\hline Amikacin & 24 & 57.1 & 0 & 0.0 & 18 & 42.9 \\
\hline Ceftazidime & 0 & 0.0 & 0 & 0.0 & 42 & 100 \\
\hline Ceftriaxone & 0 & 0.0 & 0 & 0.0 & 42 & 100 \\
\hline Ciprofloxacin & 0 & 0.0 & 0 & 0.0 & 42 & 100 \\
\hline Cefepime & 0 & 0.0 & 0 & 0.0 & 42 & 100 \\
\hline Gentamicin & 0 & 0.0 & 0 & 0.0 & 42 & 100 \\
\hline Imipenem & 1 & 2.4 & 0 & 0.0 & 41 & 97.6 \\
\hline Levofloxacin & 0 & 0.0 & 1 & 2.4 & 42 & 97.6 \\
\hline Meropenem & 1 & 2.4 & 0 & 0.0 & 41 & 97.6 \\
\hline Piperacillin & 0 & 0.0 & 0 & 0.0 & 42 & 100 \\
\hline Cotrimoxazole & 0 & 0.0 & 0 & 0.0 & 42 & 100 \\
\hline Piperacillin/tazobactam & 0 & 0.0 & 0 & 0.0 & 46 & 100 \\
\hline Tobramycin & 20 & 47.6 & 0 & 0.0 & 22 & 52.4 \\
\hline Cefoperazone/sulbactam & 2 & 4.8 & 17 & 40.5 & 23 & 54.7 \\
\hline Minocycline & 0 & 0.0 & 17 & 40.5 & 25 & 59.5 \\
\hline Tigecycline & 25 & 59.5 & 16 & 38.1 & 1 & 2.4 \\
\hline Polymyxin E & 42 & 100 & & & & \\
\hline
\end{tabular}

Table II

Distribution of the PFGE profiles of the 12 species in each unit and ward

\begin{tabular}{|l|c|c|c|c|c|c|c|c|c|c|c|c|}
\hline \multirow{2}{*}{ Wards } & \multicolumn{10}{|c|}{ Spectral type } \\
\cline { 2 - 16 } & Type A & Type B & Type C & TypeD & Type E & Type F & Type G & TypeH & Type I & TypeJ & Type K & Type L \\
\hline Intensive care unit 1 & - & - & 7 & - & - & 1 & - & - & - & - & - & 2 \\
\hline Intensive care unit 2 & 2 & 1 & 4 & - & - & - & 1 & 1 & 1 & - & 1 & - \\
\hline Intensive care unit 3 & - & - & 2 & - & 1 & - & 1 & - & - & - & - & - \\
\hline Intensive care unit 4 & - & 1 & 3 & 2 & - & - & - & 1 & - & 2 & 1 & - \\
\hline Intensive care unit 5 & - & - & 1 & 2 & - & - & - & - & - & - & - & - \\
\hline General ward 1 & - & - & - & - & - & - & - & - & - & - & - & 1 \\
\hline General ward 2 & - & - & 1 & - & - & - & - & - & - & - & - & - \\
\hline General ward 3 & - & - & 1 & - & - & - & - & - & - & - & - & - \\
\hline General ward 4 & - & - & 1 & - & - & - & - & - & - & - & - & - \\
\hline
\end{tabular}

strains containing positive ant (3')-I were sequenced; these strains contained aadA1 genes and the homology with the encoding JN814917 sequence was 100\%. PCR gene-amplified products of three strains containing positive aac(3)-I were also sequenced; these strains contained $a a c C$ genes and the homology with the encoding HQ880281 sequence was $100 \%$. PCR gene-amplified products of seven strains with positive $a a c\left(6^{\prime}\right)$ - $I b$ were sequenced; these sequences contained $a a c(6)$ - $I b$ genes and the homology with encoding JQ664644 sequence was $100 \%$. PCR gene-amplified products of two strains with positive $\operatorname{arm} A$ were sequenced; these strains contained armA genes and the homology with the encoding HQ204573 sequence was 100\%.
Conjugation test results. No successful conjugation test result was obtained in the 42 strains of the multidrug-resistant A. baumannii.

Analysis of the active efflux mechanism. After CCCP was added to imipenem, the MICs of two (4.8\%) multidrug-resistant $A$. baumannii strains to imipenem decreased four times; the MICs of the remaining strains decreased two times. After CCCP was added to ceftazidime, the MICs of two (4.8\%) multidrug-resistant A. baumannii strains to ceftazidime decreased four times; the MICs of other strains remained unchanged. After CCCP was added to meropenem, the MICs of $30(71.4 \%)$ multidrug-resistant $A$. baumannii strains to meropenem decreased four times; the MICs of 9 strains 
decreased two times. The MICs of the remaining three strains did not change. After CCCP was added to levofloxacin, the MICs of two (4.8\%) multidrug-resistant A. baumannii strains to levofloxacin decreased four times; the MICs of four strains decreased two times. The MICs of the 36 remaining strains did not change. After CCCP was added to tigecycline, the MICs of 30 multidrug-resistant A. baumannii strains to tigecycline decreased two times; 12 strains remained unchanged. Among the 42 strains, $4.8 \%$ of the multidrug-resistant $A$. baumannii strains contained active efflux mechanisms to imipenem, eftazidime, and levofloxacin. Approximately $71.4 \%$ strains exhibited active efflux mechanisms to meropenem. No active efflux mechanism was noted for tigecycline.

\section{Discussion}

A. baumannii is one of the common pathogenic bacteria causing nosocomial infections in our region (Zhao et al., 2011). This bacterium is also the main pathogenic bacteria in our ICUs, where $15.3 \%$ of the strains were isolated. Multidrug-resistant A. baumannii is the major pathogen of A.baumannii strains isolated from ICUs (Jiang et al., 2012). Immunodeficient patients in ICUs are susceptible to A. baumannii infection (Wang et al., 2010); an ICU is prone to contamination, in which sputum aspirator, ventilation, air conditioning, and infusion system can be contaminated, thereby spreading pathogens to patients (Shen et al., 2008). A. baumannii mainly causes lower respiratory tract infections (Zhang et al., 2008b; Tang et al., 2013). The 42 multidrug-resistant $A$. baumannii strains were isolated from inpatients from December 2012 to January 2013; among these strains, 39 (92.9\%) were isolated from sputum. We performed a retrospective survey for these 39 patients and found that these patients manifested symptoms of respiratory tract infection. A total of $38(90.5 \%)$ strains were isolated from the patients in ICU, suggesting that multidrug-resistant $A$. baumannii mainly causes respiratory infections in our hospital and infects patients in ICUs. This bacterium also infects patients who suffer from various acute and chronic diseases, exhibit weak immunity, receive broad-spectrum antibiotics, or undergo invasive operations.

Table II shows the PFGE profiles of 12 strains from the 42 clinical isolates of multidrug-resistant $\mathrm{A}$. baumannii that are mainly distributed in patients in ICUs. Twenty strains were type C (47.6\%), the main epidemic strain during this period in our hospital. These strains were distributed in ICUs and general wards. The same spectral type was detected in patients admitted in similar and different wards, suggesting that some of the multidrug-resistant A.baumannii strains belong to disease-causing clones. In patients carrying type $\mathrm{C}$ multidrug-resistant $A$. baumannii strains, seven strains were derived from ICU 1 , in which six of these seven patients were transferred from other hospitals. Therefore, type $\mathrm{C}$ clones may be the major epidemic strain in this area. The remaining spectral types included one to four strains, which were mainly distributed in each ICU, and only one strain was distributed in each general ward. Therefore, a part of the spectrum was distributed in our hospital.

$b l a_{\text {OXA-23 }}$ belongs to class $\mathrm{D}$ carbapenem-resistant gene, which encodes the enzyme that can hydrolyze carbapenem antibiotics; furthermore, OXA-23 is one of the major causes of multidrug resistance of $A$. baumannii (Zou et al., 2010; Yu etal., 2011). The resistance of A. baumannii to carbapenems is correlated with the production of OXA-23 carbapenemase (Mosqueda et al., 2013). Carbapenem-resistant A. baumannii producing OXA-23 is also common in the Pacific Region (Le et al., 2008). In the present study, $b l a_{\mathrm{OXA}-51}$-type carbapenemase genes were positive in 42 multidrug-resistant A.baumannii, $97.6 \%(41 / 42)$ containing bla $_{\mathrm{OXA}-23}$ -type carbapenemase genes, 100\% (42/42) containing $b l a_{\text {OXA-64 }}$ group carbapenemase gene and membrane porin gene carO, and $83.3 \%(35 / 42)$ containing $b l a_{\text {TEM }}$ $\beta$-lactamase gene. Five A. baumannii strains containing $b l a_{\mathrm{OXA}-64}$-positive gene belonging to type $\mathrm{C}$ and other different types were selected randomly for sequencing. The results showed that these strains contained $b l a_{\text {OXA-66 }}$ carbapenemase gene, in which three strains were $b l a_{\text {TEM. }}$ positive gene and considered as the $b l a_{\text {TEM-1 }}$ spectrum $\beta$-lactamase gene. This finding suggested that $b l a_{\mathrm{OXA}-23}, b l a_{\mathrm{OXA}-51}$, and $b l a_{\mathrm{OXA}-66}$ are the main carbapenemase genes in multidrug-resistant A.baumannii isolated during this period in our hospital. Culebras et al. (Culebras etal., 2010) reported an outbreak of A. baumannii producing OXA-66 in a hospital in Spain; all of the reported strains are resistant to imipenem. Zander (Zander et al., 2013) described sensitive and resistant strains in carbapenemase-resistant $A$. baumannii producing OXA-66. In this study, one multidrugresistant A. baumannii strain was sensitive to imipenem and meropenem. $b l a_{\mathrm{OXA}-66}$ and $b l a_{\mathrm{OXA}-51}$ carbapenemase genes were detected, but $b l a_{\mathrm{OXA}-23}$ carbapenemase gene was not detected. This finding suggested that the presence of bla ${ }_{\mathrm{OXA}-23}$ in multidrug-resistant A. baumannii was closely related to carbapenem resistance. Arbapenem-susceptible A. baumannii strains were also present, simultaneously producing OXA-66 and OXA-51.

Given that CCCP can significantly reduce MICs of resistant bacteria, CCCP has become an indicator of the presence of an active efflux mechanism (Rao et al., 2002). In this study, $71.4 \%$ of the strains (30/42) utilized an active efflux mechanism to meropenem; therefore, the resistance of multidrug-resistant A. baumannii to 
meropenem is related to OXA-23, OXA-51, and OXA-66 carbapenemases as well as active efflux mechanisms in a local region. Approximately $4.8 \%$ of the strains (2/42) used an active efflux mechanism to imipenem; therefore, resistance to imipenem is mainly related to OXA-23, OXA-51, and OXA-66 carbapenemases. Only $4.8 \%(2 / 42)$ of the multidrug-resistant A. baumannii strains exhibited active efflux mechanisms to eftazidime and levofloxacin. No active efflux mechanism of tigecycline was found.

In this study, plasmid-mediated quinolone resistance genes were not detected from the 42 multidrugresistant $A$. baumannii strains, indicating that the resistance of multidrug-resistant $A$. baumannii to quinolone is not related to plasmid-mediated quinolone resistance genes in our hospital.

Galimand etal. (Galimand etal., 2000) reported that bacterial resistance to aminoglycosides is due to AMES production and mutation induced by aminoglycoside antibiotics targeting the 16S rRNA gene. The $16 \mathrm{~S}$ rRNA methylase can induce drug resistance to aminoglycosides, which are commonly used in clinics (Yokoyama et al., 2003). Considering the function of AMES, researchers found more than 30 species (Chang et al., 2004). Among the 42 multidrug-resistant $A$. baumannii strains in this study, $97.7 \%$ (41/42) carried ant (3")-I gene, $81 \%(34 / 42)$ carried aac (3)-I gene, and $16.7 \%(7 / 42)$ carried aac (6)-I gene, indicating that the antimicrobial resistance to aminoglycosides of multidrug-resistant $A$. baumannii was closely related to aminoglycoside-modifying enzyme genes in our hospital. Approximately $38.1 \%$ (16/42) carried the $16 \mathrm{~S}$ rRNA gene armA; this result is lower than that in a previous study, in which $69.77 \%$ (Miao et al., 2011) of 43 A. baumannii strains are resistant to amikacin; by contrast, our result is higher than that in another study (Feng et al., 2008), in which $16 \mathrm{~S}$ rRNA methylase gene armA and $r m t \mathrm{~B}$ are not detected in 20 multidrug-resistant $A$. baumannii strains. This finding indicated that antimicrobial resistance to aminoglycosides of multidrug-resistant A. baumannii was closely related to $16 \mathrm{~S} \mathrm{rRNA}$ methylase gene $\operatorname{armA}$ in our hospital. In this study, 16 A. baumannii strains producing $16 \mathrm{~S}$ rRNA methylase gene armA were resistant to amikacin, tobramycin, and gentamicin; among these strains, one multidrug-resistant $A$. baumannii that only produced 16S rRNA methylase gene armA was also resistant to amikacin, tobramycin, and gentamicin. This finding suggested that $16 \mathrm{~S}$ rRNA methylase gene armA may simultaneously induce the resistance of A. baumannii to aminoglycosides, such as amikacin, tobramycin, and gentamicin. The 42 multidrug-resistant A. baumannii strains were resistant to gentamicin. The resistance rates to amikacin and tobramycin were $42.9 \%$ and $52.4 \%$, respectively, indicating that the resistance of multidrug-resistant A.baumannii to gentamicin is closely related to ant (3")-I gene and AAC (3)-I gene. Seven multidrug-resistant A.baumannii strains with $A A C$ (6)-I positive are resistant to tobramycin, including one sensitive strain and six resistant strains to amikacin. This result suggested that the multidrug resistance of $A$. baumannii to tobramycin is closely related to $a a c$ (6)-I genes. Among the 42 multidrug-resistance A. baumannii strains in this study, 41 carried the qac $\mathrm{E} \Delta 1$ gene, and the positive rate was $97.7 \%$. Such a high qacE $\Delta 1$ gene carrying rate should alert our hospital disinfection department, and bacterial resistance to disinfectants may be one of the important factors influencing nosocomial infections.

In conclusion, clinical isolates of multidrug-resistant A. baumannii with type $\mathrm{C}$ clones were the predominant strains from December 2012 to January 2013 in our hospital, and there existed clonal dissemination in nosocomial multidrug-resistant A. baumannii. Furthermore, multidrug-resistant A. baumannii is closely related to various resistance genes. All of these highlight the importance of understanding the status of drugresistance and local specificities in multidrug-resistant A. baumannii for antimicrobial empirical therapy and for implementing.

Disclosure Statement. The authors have no conflict of interest.

\section{Literature}

Chang L.L., H.F. Chen and W.J. Wu. 2004. Contribution of integrons, and SmeABC and SmeDEF efflux pumps to multidrug resistance in clinical isolates of Stenotrophomonas maltophilia (in Chinese). J. Antimicrob. Chemother. 53: 518-521.

Culebras E., F. González-Romo and J.J. Picazo. 2010. Outbreak of Acinetobacter baumannii producing OXA-66 in a Spanish hospital: epidemiology and study of patient movements. Microb. Drug. Resist. 16: 309-315.

Feng G.Z., Y. Zhang and S.D. Zhao. 2008. Study on the genes of $16 \mathrm{~S} r$ RNA methylase and a minoglycoside modifying enzyme in multi-resistant Acinetobacter baumannii (in Chinese). Chin. J. Infect. Chemother. 8: 303-306.

Galimand M., G. Gerbaud and P. Courvalin. 2000. Spectinomycin resistance in Neisseria spp. due to mutations in 16S rRNA. Antimicrob. Agents. Chemother. 44: 1365-1366.

Hu F.M., D.M. Zhu and P.C. Wu. 2008. The molecular characteristics of E.coli and Klebsiella spp.with CLSI ESBL-screening test positive but cefepime-susceptible (in Chinese). Chin. J. Lab. Med. 31: $1128-1133$.

Jiang M.J. 2012. Drug resistance and distribution in common clinical Gram-negative bacilli collected from intensive care units (in Chinese). Chin. J. Exp. Clin. Infect. Dis. (Electronic Edition) 6: 42-45. Le Hello. S., V. Falcot and T. Naas. 2008. Molecular epidemiology of carbapenem-resistant Acinetobacter baumannii in New Caledonia. Clin. Microbiol. Infect. 14, 977-978.

Miao X.J., Z.L. Chen and Y. Li. 2011. Study on 16S rRNA methylase genes in 134 Acinetobacter baumannii strains (in Chinese). Chinese Journal of Nosocomiology 21: 1075-1077.

Montero, J. Gómez, J.P. Horcajada, J. Vila and others. 2013. Globally expanding carbapenemase finally bursts in Spain: Nosocomial 
outbreak of Acinetobacter baumannii producing a plasmid-encoded OXA-23 in Barcelona, Spain. Antimicrob. Agents. Chemother. 57: 5155-5157.

Mosqueda N., P. Espinal, C. Cosgaya, S. Viota, V. Plasensia, F.A. Lerma, M. Park C.H., A. Robicsek and D.C. Hooper. 2006. Prevalence in the United States of $a a c\left(6^{\prime}\right)-I b$-cr encoding a ciprofloxacinmodifying enzyme. Antimicrob. Agents. Chemother. 50: 3953-3955. Rao Y., Z.L. Zeng and Z.L. Chen. 2002. Active efflux mechanism of antibiotic resistance. World Notes On Antibiotics 23: 109-114.

Shen C.H. and M. Ou. 2008. Clinical distribution and drug resistance of 122 strains of Acinetobacter baumannii (in Chinese). Lab. Med. Clin. 5: 853-854.

Shen J.L., D.M. Zhu and M.G. Wang. 2008. The relationship between acquired carbapenemases and resistance of gram-negative bacilli (in Chinese). Chin. J. Lab. Med. 31: 408-414.

Tang X.L. and J. Yang. 2013. Analysis of infection status and drug resistance of Acinetobacter baumannii in ICU (in Chinese). Chong. Qing. Medcine 42: 302-303.

Wang L.Y. and W.X. Huang. 2010. Prevention Progress of Panresistant Acinetobacter baumannii (in Chinese). ChongQing Medicine 39: 2808-2811.

Wang W., J.F. Mao and W.Z. Xu. 2009. Drug-resistance and Disinfectant-resistant Genes of Chlorhexidine-sulfadiazine-resistant Escherichia coli Isolates (in Chinese). Chinese Journal of Nosocomio$\log y$ 19: 247-249.

Wang Y.M. and J.L. Shen. 2012. Primary study on efflux pump mechanism in multidrug-resistant Acinetobacter baumannii (in Chinese). Acta Universitatis Medicinalis Anhui 47: 38-40.

Yang H., H. Wang and Y.C. Xu. 2008. The mechanism study of plasmid-mediated quinolone resistance in clinical isolates of Enterobacteriaceae from nine teaching hospitals in China (in Chinese). Chin. J. Lab. Med. 31: 969-974.
Yang Y.M., H.F. Ye and X.M. Zhou. 2011. Detection of New Delhi metallo- $\beta$-lactamase I gene in Klebsiella ozaenae and Acinetobacter baumannii (in Chinese). Int. J. Lab. Med. 32: 1407-1409.

Yokoyama K., Y. Doi and Y. Arakawa. 2003. Acquisition of 16S rRNA methylase gene in Pseudomonas aeruginosa. Lancet 362:1888-1893

Yu L., F.R. Jiang and D.H. Su. 2011. Study on the genotypes of OXA-23 and qacE'1 in multidrug-resistant Acinetobacter baumannii (in Chinese). Lab. Med. Clin. 15: 1793-1797.

Zander E., A. Chmielarczyk and P.G. Higgins. 2013. Conversion of OXA-66 into OXA-82 in clinical Acinetobacter baumannii isolates and association with altered carbapenem susceptibility. J. Antimicrob. Chemother. 68: 308-311.

Zhang R., J.C. Cai and G.X. Chen. 2008a. Reduced carbapenem susceptibility in Klebsiella pneumoniae clinical isolates is due to combination of $\beta$-lactamases and the loss of OmpK36 porin (in Chinese). Chinese Journal of Microbiology And Immunology 28: 44-49. Zhang X.B., X.L. Gong and W.L. Fu. 2008b. Analysis of Clinical Distribution and Drug-resistance of Acinetobacter baumannii (in Chinese). Chinese Journal of Nosocomiology 18: 428-430.

Zhao S.P., M.J. Jiang and G.M. Wang. 2011. Distribution and Antimicrobial Resistance of Commonly Encountered Gram-negative Bacilli in Clinical in Taian region (in Chinese). Chin. J. Clinicians. (Electronic Edition) 5: 179-181.

Zhi Z.Q., Z.H. He and H.F. Zou. 2005. The study on genotyping of $\beta$-lactamases and aminoglycoside-modifying enzyme in multiresistant Pseudomonas aeruginosa (in Chinese). Chin. J. Lab. Med. 28: 1121-1124.

Zou J.M., A.P. Zhang and S.J. Deng. 2010. An infection outbreak of OXA-23 carbapenemase-producing imipenem-resistant Acinetobacter baumannii (in Chinese). Chinese Journal Of Infection Control 9: 235-237. 
